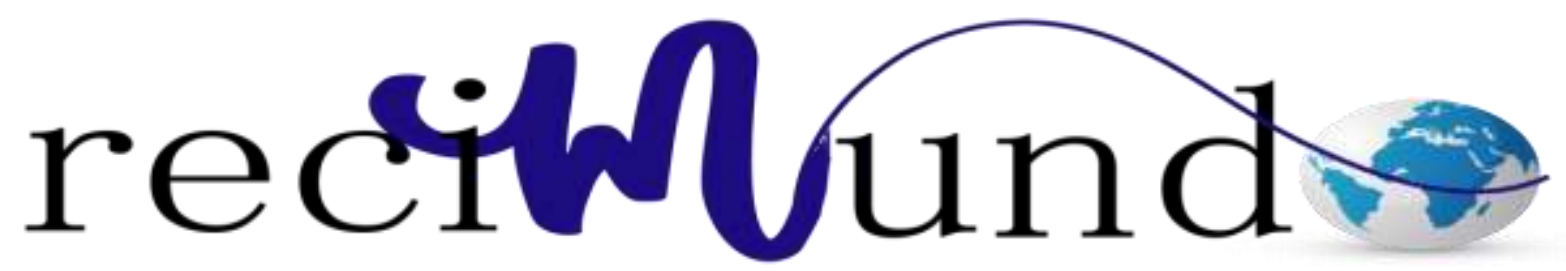

Revista Científica Mundo de la Investigación y el Conocimiento

\author{
María Victoria Peñafiel Rodríguez a ; Sandra Marcela Quisiguiña Guevara ${ }^{\text {b }}$; Carlos \\ Alberto Alban Hurtado ${ }^{c}$; Hernán Ramiro Robalino Campos ${ }^{\mathrm{d}}$
}

Comparación de la resistencia a la fuerza de compresión de las resinas híbrida, nanohíbrida y bulk fill

Comparison of the resistance of the dihybrid, hybrid and Bulk Fill resins to the compression force

Revista Científica Mundo de la Investigación y el Conocimiento. Vol. 3 núm.3, septiembre, ISSN: 2588-073X, 2019, pp. 585-595

DOI: $10.26820 /$ recimundo/3.(3).septiembre.2019.585-595

URL: http://recimundo.com/index.php/es/article/view/539

Código UNESCO: 3213.13 Estomatología

Tipo de Investigación: Artículo de Revisión

Editorial Saberes del Conocimiento

Recibido: 15/05/2019

Aceptado: 23/06/2019

Publicado: 30/09/2019

Correspondencia: maríavictoria5952@gmail.com

a. Odontóloga; Universidad Nacional de Chimborazo; Riobamba, Ecuador; maríavictoria5952@gamil.com

b. Especialista en Estética y Operatoria Dental; Odontóloga; Universidad Nacional de Chimborazo Riobamba, Ecuador; smquisiguina@unach.edu.ec

c. Especializacao em Proteses Dentaria; Especializacao em Endodontia; Odontólogo; Universidad Nacional de Chimborazo; Riobamba, Ecuador; caalban@unach.edu.ec

d. Ingeniero en Comercio Exterior mención en Negociaciones Internacionales; Riobamba, Ecuador; ramiro9rch@hotmail.com 


\section{Comparación de la resistencia a la fuerza de compresión de las resinas híbrida, nanohíbrida y bulk fill}

Vol. 3, núm. 3., (2019)

María Victoria Peñafiel Rodríguez; Sandra Marcela Quisiguiña Guevara; Carlos Alberto Alban Hurtado; Hernán Ramiro Robalino Campos

\section{RESUMEN}

Objetivo: Comparar la resistencia de las resinas híbrida, nanohíbrida y Bulk Fill a la fuerza de compresión mediante estudios de laboratorio para identificar la resina que posee mejor resistencia compresiva en el Centro de Fomento Productivo Metalmecánico Carrocero. Materiales y Métodos: Se realizó un estudio observacional, comparativo, in vitro experimental y transversal, se designó 10 probetas cilíndricas para los tres grupos con medidas de 4x4mm establecidas en la norma ISO 4049. Las resinas utilizadas fueron la Filtek Z250 3M, Filtek Z350 XT 3M y Filtek Bulk Fill 3M. Resultados: Los datos obtenidos de resistencia fueron 162,998 MPa para la Filtek Z250, 177,05 MPa para la Filtek Z350 XT y 172,305 MPa para la Filtek Bulk Fill. Conclusión: Los análisis estadísticos determinaron una distribución normal de datos, el análisis de varianzas (ANOVA) no determinó conclusiones definitivas en razón de que no hubo suficiente evidencia, sin embargo los estadísticos descriptivos demostraron que la resina con mayor resistencia fue la nanohíbrida representada por la Filtek Z350 XT.

Palabras Claves: Resistencia a la Fuerza de Compresión; Resina Híbrida; Resina Nanohíbrida; Resina Bulk Fill. 


\section{Comparación de la resistencia a la fuerza de compresión de las resinas \\ híbrida, nanohíbrida y bulk fill}

Vol. 3, núm. 3., (2019)

María Victoria Peñafiel Rodríguez; Sandra Marcela Quisiguiña Guevara; Carlos Alberto Alban

Hurtado; Hernán Ramiro Robalino Campos

\section{ABSTRACT}

Aim: o compare the resistance of the hybrid, nanohybrid and Bulk Fill resins to the compression force by means of laboratory studies to identify the resin that has the best compressive strength at the Centro de Fomento Productivo Metalmecánico Carrocero. Materials and Methods: An observational, comparative, in vitro - experimental and cross-sectional study was carried out, 10 cylindrical specimens were designated for the three groups with $4 \times 4 \mathrm{~mm}$ measurements established in ISO 4049. The resins used were Filtek Z250 3M, Filtek Z350 XT 3M and Filtek Bulk Fill 3M. Results: The data obtained for resistance were 162,998 MPa for the Filtek Z250, 177.05 MPa for the Filtek Z350 XT and 172,305 MPa for the Filtek Bulk Fill. Conclusion: The statistical analyzes determined a normal distribution of data, the analysis of variances (ANOVA) did not determine definitive conclusions because there was not enough evidence, however the descriptive statistics showed that the resin with greater resistance was the nanohybrid represented by the Filtek Z350 XT.

Key Words: Compressive Strength; Hybrid Resin; Nanohybrid Resin; Bulk Fill Resin. 


\section{Comparación de la resistencia a la fuerza de compresión de las resinas híbrida, nanohíbrida y bulk fill}

Vol. 3, núm. 3., (2019)

María Victoria Peñafiel Rodríguez; Sandra Marcela Quisiguiña Guevara; Carlos Alberto Alban Hurtado; Hernán Ramiro Robalino Campos

\section{Introducción.}

La odontología en los últimos años ha experimentado muchos cambios y avances tanto en sus procedimientos como en sus materiales, uno de ellos es la introducción de varios compuestos usados en la restauración dental, como las resinas dentales de fotocurado, estas en el transcurso del tiempo han suplantado a las amalgamas, las cuales se utilizaban para restauraciones dentales en generaciones pasadas. (1)

Las resinas de fotocurado son muy convenientes y aceptables por sus propiedades físico químicas, por la estética que presentan y por los procesos de polimerización. (2) Las propiedades de las resinas compuestas en sí, están directamente relacionadas con la composición de la porción orgánica que contiene, la cantidad y calidad de la carga y la técnica que es utilizada en la fotopolimerización de esta. (3)

Sin embargo, es importante recalcar que, a pesar de tener varios aspectos positivos, las resinas generan también claras desventajas; una de ellas es el grado de compresión que sufren cuando se realiza el proceso de polimerización, por lo cual es desfavorable, ya que el éxito de la restauración dental disminuye. (2)

De esta manera el grado de compresión se convierte en uno de los principales problemas del fracaso de una restauración dental, porque causa daños clínicos como fractura de la restauración, fuga marginal, sensibilidad postoperatoria y recurrencia de caries secundaria en el diente previamente restaurado. (2)

Por tal motivo al haber un fracaso de la restauración, se tiene que dar un reemplazo 


\section{Comparación de la resistencia a la fuerza de compresión de las resinas \\ híbrida, nanohíbrida y bulk fill}

Vol. 3, núm. 3., (2019)

María Victoria Peñafiel Rodríguez; Sandra Marcela Quisiguiña Guevara; Carlos Alberto Alban

Hurtado; Hernán Ramiro Robalino Campos

inmediato de la restauración dental fallida, lo que sigue siendo el problema más común en odontología general y sobre todo en operatoria dental, donde se realiza un mayor número de tratamientos restauradores en personas adultas, aumentando el gasto económico del paciente. (4)

En varios estudios, la caries secundaria constituye en una de las razones principales para que se sustituyan restauraciones dentales. En la publicación de Soncini et al, citado por Pallesen et al (4) muestra varias restauraciones oclusales con resina compuesta en niños de 6 a 10 años, todo esto en un período de 3 años, obteniéndose como resultado que de las 753 restauraciones, $112(14.9 \%)$ son reemplazadas y $21(2.8 \%)$ son reparadas. Se menciona además que varias de las razones por la que más se reemplaza dichas restauraciones son la caries secundaria en un 52\% y la fractura de la restauración en un $2 \%$. (4)

Conociendo los problemas que conlleva una restauración dental fracasada se han realizado estudios con el fin de aportar características que ayuden al éxito de una restauración centrándose en las propiedades que una resina dental debe poseer. En Perú, en un estudio llevado a cabo en el año 2017 se indica que con el pasar del tiempo, la industria odontológica se ha enfocado en la mejora de materiales de restauración dental, realizando modificaciones en la fase orgánica, inorgánica y en los procesos de fotopolimerización del material restaurador. (5)

Varios fabricantes mundiales se han centrado en la resistencia a la compresión que una resina debe tener, porque optimizando esta propiedad el material puede resistir mejor a las fuerzas verticales durante el acto masticatorio o en movimientos parafuncionales. La mayoría de las fuerzas transmitidas en la región posterior de la cavidad bucal son específicamente 


\section{Comparación de la resistencia a la fuerza de compresión de las resinas híbrida, nanohíbrida y bulk fill}

Vol. 3, núm. 3., (2019)

María Victoria Peñafiel Rodríguez; Sandra Marcela Quisiguiña Guevara; Carlos Alberto Alban Hurtado; Hernán Ramiro Robalino Campos

compresivas, por esta razón dichas fuerzas pueden ocasionar la fractura de la restauración dental y del órgano dentario. (5)

En Ecuador se han realizado estudios de investigación de varios tipos de resina sobre la resistencia a la compresión obteniéndose como resultados, que las resinas compuestas de microrelleno no muestran una significancia en la tenacidad a la fractura, al contrario que la resina hibrida que si indica una disminución considerable a la fractura. En este estudio también se evalúa a una resina de tipo Bulk Fill, este producto muestra una resistencia compresiva muy alta, pese a que este material es aplicado y fotocurado en un incremento, resultando tener valores superiores en resistencia en comparación con los otros materiales de restauración. (6)

Por lo mencionado es necesario que se realicen análisis sobre las propiedades físicas mecánicas del material restaurador con el propósito de evitar el fracaso de dichos tratamientos.

\section{Materiales y métodos.}

Se aplicó un estudio observacional, comparativo, in vitro - experimental y transversal, las técnicas fueron la observación y encuesta y los instrumentos la bitácora y el cuestionario respectivamente. La encuesta fue aplicada a los estudiantes de Clínica Integral y determino que la marca de resina más adecuada para realizar las probetas de estudio fue la 3M ESPE, se elaboró 10 probetas cilíndricas para los tres grupos bajo las instrucciones del fabricante con medidas de 4x4mm establecidas en la norma ISO 4049. Las resinas utilizadas fueron la Filtek Z250 3M como híbrida, Filtek Z350 XT 3M como nanohíbrida y Filtek Bulk Fill 3M como Bulk Fill. Las muestras fueron sometidas a pruebas de resistencia a la compresión en la máquina universal en el 


\section{Comparación de la resistencia a la fuerza de compresión de las resinas \\ híbrida, nanohíbrida y bulk fill}

Vol. 3, núm. 3., (2019)

María Victoria Peñafiel Rodríguez; Sandra Marcela Quisiguiña Guevara; Carlos Alberto Alban

Hurtado; Hernán Ramiro Robalino Campos

Laboratorio de Resistencia de Materiales del Centro de Fomento Productivo Metalmecánico Carrocero del Honorable Gobierno Provincial de Tungurahua. Los resultados fueron analizados mediante el SPSS mediante pruebas de normalidad, ANOVA, estadístico de Levene y estadísticos descriptivos.

\section{Resultados.}

Se obtuvo valores sobre la fuerza máxima y la fuerza de fluencia en newtons $(\mathrm{N})$ y el esfuerzo máximo de compresión en megapascales (MPa). La resina Filtek Z250 obtuvo medias de 2048,308 N, 62,481 N y 162,998 MPa respectivamente, mientras que la resina Filtek Z350 XT alcanzo medias de 2224,8811 N, 92,0356 N y 177,05 MPa respectivamente y la Filtek Bulk Fill anoto valores sobre la fuerza máxima de 2165,382 N, la fuerza de fluencia como 188,074 N y el esfuerzo máximo de compresión valores de 172,305 MPa. Con los datos mencionados se estableció la comparación de la resistencia a la fuerza de compresión entre los tres grupos mediante pruebas de normalidad resultando una distribución normal en los datos, en el análisis de varianzas ANOVA y el estadístico de Levene no existió suficiente evidencia para encontrar diferencias entre los grupos de estudio. Sin embargo se determinó en los estadísticos descriptivos que el grupo de resina que obtuvo mayor resistencia a la compresión fue la resina nanohíbrida representada por la Filtek Z350 XT.

\section{Discusión.}

En el presente estudio se compara la resistencia de las resinas híbrida, nanohíbrida y Bulk Fill a la fuerza de compresión mediante pruebas de laboratorio. Obteniendo resultados sobre la 


\section{Comparación de la resistencia a la fuerza de compresión de las resinas híbrida, nanohíbrida y bulk fill}

Vol. 3, núm. 3., (2019)

María Victoria Peñafiel Rodríguez; Sandra Marcela Quisiguiña Guevara; Carlos Alberto Alban Hurtado; Hernán Ramiro Robalino Campos

fuerza máxima de la resina Filtek Z250 3M una media de 2048,308 N, la resina Filtek Z350 XT

3M una media de 2224,881 N y la resina Filtek Bulk Fill 3M una media de 2165,382 N. Sobre la fuerza de fluencia la resina Filtek Z250 3M tiene una media de 62,481 N, la resina Filtek Z350 XT 3M una media de 92,0356 N y la Filtek Bulk Fill 3M una media de 188,074 N. Con los siguientes datos se establece una comparación con el estudio realizado por Guerra (7) en el cual menciona la resistencia a la compresión entre la resina Filtek Z350 XT con técnica incremental y la Bulk Fill con técnica monoincremental, respecto a la fuerza máxima la Z350 XT obtuvo 2584,80 N mientras que la Bulk Fill 2402,67 N. Se puede notar que los valores reportados de la resina Z350 XT con respeto a la fuerza máxima tienen concordancia con los valores obtenidos en este estudio, al contrario de la resina Bulk Fill en la cual los valores no coinciden.

En los datos recopilados en esta investigación sobre el esfuerzo máximo de compresión medido en MPa, la resina Filtek Z250 3M tiene una media de 162,998 MPa, la Filtek Z350 XT 3M una media de 177,05 MPa y la resina Filtek Bulk Fill 3M una media de 172,305 MPa. En Perú, en un trabajo de investigación publicado en el año 2018, comparan la resistencia a la compresión y flexión de las resinas Bulk Fill (Opus ${ }^{\mathrm{TM}}$ Bulk Filk, Tetric ${ }^{\circledR}$ N-Ceram Bulk Fill y Filtek $^{\mathrm{TM}}$ Bulk Fill) y la resina Filtek Z350 XT, en el cual las resinas Bulk Fill presentan mayor resistencia, a pesar de ser resinas monoincrementales las mismas que utilizan diferentes sistemas para iniciar la fotoiniciacion. La resina que obtiene mayor resistencia es la Filtek ${ }^{\mathrm{TM}}$ Bulk Fill con una media de 235.59 MPa y la más baja la Filtek Z350 XT con 99.28 MPa. (8) Estos datos no coinciden con la presente investigación, ya que según los resultados arrojados, la resina Z350 XT tiene mejor resistencia a la compresión que la resina Bulk Fill. 


\section{Comparación de la resistencia a la fuerza de compresión de las resinas \\ híbrida, nanohíbrida y bulk fill}

Vol. 3, núm. 3., (2019)

María Victoria Peñafiel Rodríguez; Sandra Marcela Quisiguiña Guevara; Carlos Alberto Alban

Hurtado; Hernán Ramiro Robalino Campos

En el estudio realizado por Garzón (9) sobre la resistencia a la compresión de la resina Filtek P60 3M polimerizada con lámpara LED, se encuentran resultados muy variables, el grupo de control tiene una resistencia media de $111 \mathrm{MPa}$, mientras que el grupo en el que el material es polimerizado con calor y nitrógeno se encuentran valores de 167,97 $\mathrm{MPa}$, sin embargo no coindicen con los valores que menciona la descripción de la casa comercial, en la que la resistencia compresiva es de $350 \mathrm{MPa}$ a $400 \mathrm{MPa}$. La resina P60 es de la casa comercial 3M al igual que las resinas utilizadas en este estudio, por esto se puede establecer semejanzas en sus valores, pero dichos datos se acercan solamente a los de la resina Filtek Z250 con un resistencia media de 162,97 MPa.

Falcón et al (5) para comparar la resistencia a la compresión entre la resina Filtek Z250 y la resina SonicFill, utiliza probetas de $4 \times 4 \mathrm{~mm}$ y las somete a pruebas de laboratorio, se obtiene resultados de 289,7 MPa para la Filtek Z250 y 257,73 MPa para la SonicFill. Se compara los resultados de las probetas por ser las mismas medidas utilizadas en este estudio, y dichos valores están por debajo con una diferencia aproximadamente de $87 \mathrm{MPa}$.

De igual forma se han realizado estudios sobre resistencia a la compresión pero en órganos dentales, este es el caso de Millingalli (10) que realiza una investigación en premolares, para determinar la compresión de dos resinas de la casa comercial 3M específicamente la Filtek Z350 XT y la Filtek Bulk Fill Fluida y de dos resinas de la casa comercial VOCO entre las que están la Grandio y la Grandio Flow. Las resinas 3M alcanzan en promedio 305,32 MPa y las VOCO 277,58 MPa. Ninguno de los dos valores se asemeja a los de este estudio, concluyendo 


\section{Comparación de la resistencia a la fuerza de compresión de las resinas híbrida, nanohíbrida y bulk fill}

Vol. 3, núm. 3., (2019)

María Victoria Peñafiel Rodríguez; Sandra Marcela Quisiguiña Guevara; Carlos Alberto Alban Hurtado; Hernán Ramiro Robalino Campos

que uno de los factores para que los resultados infieran significativamente, sea por que las muestras fueron manipuladas directamente en el órgano dentario y no en probetas.

\section{Conclusiones.}

Con los datos mencionados se estableció la comparación de la resistencia a la fuerza de compresión entre los tres grupos mediante pruebas de normalidad y se concluyó una distribución de datos normales, en el análisis de varianzas ANOVA y el estadístico de Levene se determinó que no hay suficiente evidencia para encontrar diferencias entre los grupos de estudio, esto se comprobó debido a que los valores de significancia fueron mayores a 0,05 ( $\mathrm{p}=0,817)$. Sin embargo se determinó en los estadísticos descriptivos que el grupo de resina que obtuvo mayor resistencia a la compresión fue la resina nanohíbrida representada por la Filtek Z350 XT.

\section{Conflicto de intereses.}

Los autores no manifiestan ningún conflicto de interés.

\section{Agradecimientos.}

Los autores agradecen a los estudiantes de Clínica Integral de la Carrera de Odontología de la Universidad Nacional de Chimborazo y al personal del Centro de Fomento Productivo Metalmecánico Carrocero del Honorable Gobierno Provincial de Tungurahua.

\section{Bibliografía.}

1. Germscheid W, Gosse De Gorre L, Sullivan B, O’Neill C, Price RB, Labrie D. Post-curing in dental resin-based composites. Dent Mater [Internet]. 2018;34(9):1367-77. Available from: 


\section{Comparación de la resistencia a la fuerza de compresión de las resinas \\ híbrida, nanohíbrida y bulk fill}

Vol. 3, núm. 3., (2019)

María Victoria Peñafiel Rodríguez; Sandra Marcela Quisiguiña Guevara; Carlos Alberto Alban

Hurtado; Hernán Ramiro Robalino Campos

https://doi.org/10.1016/j.dental.2018.06.021

2. Park J, Lee G, Kim J, Park M, Ko C, Kim H-I, et al. Polymerization shrinkage, flexural and compression properties of low-shrinkage dental resin composites. Dent Mater J. 2014;33(1):104-10.

3. Almeida-Chetti VA, Macchi RL, Iglesias ME. Effect of Post-Curing treatment on mechanical properties of composite resins. Acta Odontológica Latinoam. 2014;27(2):72-6.

4. Pallesen U, Van Dijken JW, Halken J, Hallonsten A-L, Höigaard R. A prospective 8-year follow-up of posterior resin composite restorations in permanent teeth of children and adolescents in Public Dental Health Service: reasons for replacement. Clin Oral Invest. 2013;1-9.

5. Falcón Cabrera G, Benavente Acurio P. Comparación de la resistencia compresiva de resinas convencionales vs resinas tipo bulk fill. 2017.

6. Vélez Cuenca TM. Resistencia de la resina convencional (nanohíbrida) y resina Bulk-Fill a la fractura con técnicas incremental y monoincremental. Estudio comparativo in-vitro. 2016.

7. Guerra Midolo DD. Resistencia a la compresión entre una resina con técnica incremental Filtek Z350 XT de 3M ESPE y una monoincremental Filtek Bulk Fill de 3M ESPE. Estudio In Vitro, Arequipa, 2017. 2017.

8. Borja Farfán NE, Loyola Livias OD. Comparación in vitro de la resistencia a la compresión y resistencia flexural de resinas Bulk Fill (Opus TM Bulk Fill, Tetric ${ }^{\circledR}$ N-Ceram Bulk Fill y Filtek TM Bulk Fill). 2019.

9. Garzón Flores JE. Estudio comparativo de resistencia a la compresión de la resina Filtek P60 3M sometida a calor y presión; Estudio In Vitro. 2016.

10. Millingalli Vega HS. Determinar la resistencia a la compresión vertical en cuatro resinas de nanotecnología de dos casas comerciales en técnica cominada entre resina fluida y convencional a través de la técnica incremental en restauraciones clase II ocluso-distal. 2016. 